ARTICLE

Received 9 May 2014 | Accepted 23 Jul 2014 | Published 4 Sep $2014 \quad$ DOI: 10.1038/ncomms5786

\title{
Widespread transient Hoogsteen base pairs in canonical duplex DNA with variable energetics
}

Heidi S. Alvey¹, Federico L. Gottardo', Evgenia N. Nikolova² \& Hashim M. Al-Hashimi ${ }^{3}$

Hoogsteen $\left(H G\right.$ ) base pairing involves a $180^{\circ}$ rotation of the purine base relative to WatsonCrick (WC) base pairing within DNA duplexes, creating alternative DNA conformations that can play roles in recognition, damage induction and replication. Here, using nuclear magnetic resonance $R_{1 p}$ relaxation dispersion, we show that transient HG base pairs occur across more diverse sequence and positional contexts than previously anticipated. We observe sequencespecific variations in $\mathrm{HG}$ base pair energetic stabilities that are comparable with variations in WC base pair stability, with HG base pairs being more abundant for energetically less favourable WC base pairs. Our results suggest that the variations in HG stabilities and rates of formation are dominated by variations in WC base pair stability, suggesting a late transition state for the WC-to-HG conformational switch. The occurrence of sequence and position-dependent HG base pairs provide a new potential mechanism for achieving sequence-dependent DNA transactions.

\footnotetext{
${ }^{1}$ Department of Chemistry and Biophysics, University of Michigan, 930 N. University Avenue, Ann Arbor, Michigan 48109, USA. ${ }^{2}$ Department of Molecular Biology, The Scripps Research Institute, 10550 North Torrey Pines Road, La Jolla, California 92037, USA. ${ }^{3}$ Department of Biochemistry and Chemistry, Duke University School of Medicine, 307 Research Dr, Nanaline H. Duke Building, Durham, North Carolina 27710, USA. Correspondence and requests for materials should be addressed to H.M.A.-H. (email: hashim.al.hashimi@duke.edu).
} 
W e recently showed ${ }^{1-3}$ using nuclear magnetic resonance (NMR) $R_{1 \rho}$ relaxation dispersion $(\mathrm{RD})^{4-6}$ that $\mathrm{A} \bullet \mathrm{T}$ and $\mathrm{G} \bullet \mathrm{C}$ Watson-Crick (WC) base pairs (bps) in CA/TG and TA/TA steps of duplex DNA transiently form Hoogsteen (HG) $\mathrm{bps}^{7}$ with populations ranging between 0.14 and $0.49 \%$ and lifetimes between 0.3 and $2.5 \mathrm{~ms}$ at $\mathrm{pH} \sim 6$ (Fig. 1a). HG bps form through $180^{\circ}$ rotation of the purine base around the glycosidic bond from an anti to syn conformation (Fig. 1a). HG bps modify the structural and chemical presentation of DNA and thereby can play unique roles (reviewed in ref. 8) in DNA-protein recognition ${ }^{9-12}$, damage induction ${ }^{13}$ and repair $^{14,15}$ as well as replication ${ }^{16,17}$. For example, by narrowing the minor groove, HG bps have been shown to alter the DNA electrostatic potential 'seen' by DNA-binding proteins at the bp edges in the DNA grooves in the context of p53-DNA complexes $^{12,18,19}$. HG bps can also transiently expose DNA sites that are otherwise inaccessible in WC bps, and thereby provide new mechanisms for damage induction. For example, using computational mapping, Bohnuud et al. ${ }^{13}$ recently showed that transient $\mathrm{G} \bullet \mathrm{C}^{+} \mathrm{HG}$ bps could explain the susceptibility and accessibility of cytosines to hydroxymethylation by formaldehyde, thus explaining a long-standing mystery that has persisted for over 25 years. There is also structural and biochemical evidence that some members of the low-fidelity Y-family polymerases replicate DNA using HG pairing as the dominant mechanism, thus providing a mode for bypassing a variety of lesions on the WC face during replication ${ }^{16,17,20}$. Naked duplexes consisting entirely of HG bps have been reported in A-T-rich sequences that form parallel ${ }^{21}$ and anti-parallel stranded 22,23 DNA. Computational studies on duplexes also suggest that the HG bp is a reasonable conformation, only slightly less stable than the canonical WC bp ${ }^{24,25}$.

Considering that $\mathrm{HG}$ bps are an energetically favourable alternative to $\mathrm{WC}$ bps that can provide new mechanisms in a wide variety of DNA biochemical processes, it is of great interest to explore whether the transient HG bps observed by NMR are confined to flexible CA and TA steps, or occur more broadly across distinct sequence and position contexts in duplex DNA. Likewise, it is of interest to examine the sequence specificity of transient HG bp formation as this could provide new mechanisms for achieving sequence-specific DNA transactions that are based on shape ${ }^{19}$.

By carrying out ${ }^{13} \mathrm{C}$ and ${ }^{15} \mathrm{~N} R_{1 \rho}$ NMR RD measurements on 33 bps in eight distinct canonical DNA duplexes, we show that transient HG bps are not limited to CA and TA steps, but rather occur broadly across diverse sequence and positional contexts. We find that both the energetic stability and rates of $\mathrm{HG} b \mathrm{p}$ formation exhibit a dependence on sequence and position, with HG bps forming faster and being more abundant in energetically less favourable WC bps.

\section{Results}

Widespread transient HG bps in duplex DNA. To more broadly examine the occurrence and sequence specificity of transient $\mathrm{HG}$ bps in canonical duplex DNA, we carried out ${ }^{13} \mathrm{C}$ and ${ }^{15} \mathrm{~N} R_{1 \rho}$ NMR RD measurements targeting sugar $\mathrm{C}^{\prime}$ and base $\mathrm{C} 6 / 8$ or $\mathrm{N} 1 / 3$ resonances in $20 \mathrm{~A} \bullet \mathrm{T}$ and $13 \mathrm{G} \bullet \mathrm{C}$ bps in eight DNA duplexes that encompass a variety of sequence motifs, including $(\mathrm{A} \bullet \mathrm{T})_{n}$ repeats of varying length $(n=2,4,5$ and 6$)$, a $(\mathrm{CA})_{3}$ repeat, a duplex sequence that forms $\mathrm{HG}$ bps on binding to the antibiotic echinomycin, ${ }^{1,26,27}$ a $(C G)_{3}$ repeat capable of forming $\mathrm{Z}_{-} \mathrm{DNA}^{28}$, and a $\mathrm{B} / \mathrm{Z}$ junction forming sequence ${ }^{29,30}$ (Fig. 1b). The targeted bps (Fig. 1b, highlighted in stars) encompass $6 / 10$ dinucleotide steps that are positioned $1-6$ bps away from the closest terminal end. $R_{1 \rho} \mathrm{RD}$ experiments were performed at $\mathrm{pH}$
5.2-7.5 and 5.2-5.4 for $\mathrm{A} \bullet \mathrm{T}$ and $\mathrm{G} \bullet \mathrm{C}$ bps, respectively. We use low-pH conditions for transient (and protonated) $\mathrm{G} \bullet \mathrm{C}$ bps in order to increase the WC-to-HG chemical exchange signature detected by NMR $R_{1 \rho} \mathrm{RD}^{3}$. We recently reported a detailed analysis of the $\mathrm{pH}$ dependence of chemical exchange corresponding to transient $\mathrm{HG}$ bp formation and how measurements at such lower $\mathrm{pH}$ conditions can be qualitatively interpolated to assess exchange at higher $\mathrm{pH}^{3}$.

The $R_{1 \rho}$ NMR RD experiment measures the line broadening contribution to resonances of interest due to chemical exchange with a transient, lowly populated species ${ }^{4,5}$. In all cases, we measured significant ${ }^{13} \mathrm{C}$ and/or ${ }^{15} \mathrm{~N} R_{1 \rho}$ RD (Supplementary Table 1) consistent with chemical exchange (Fig. 1c and Supplementary Fig. 1). A two-state analysis $\left(\mathrm{A} \underset{k_{\mathrm{B}}}{\stackrel{k_{\mathrm{A}}}{\rightleftarrows}} \mathrm{B}\right)$ of the $R_{1 \rho}$ data $^{4-6}$ (see Methods) yielded populations $\left(p_{\mathrm{B}}=\sim 0.08\right.$ $2.73 \%)$ and lifetimes $\left(\tau_{\mathrm{B}}=\sim 0.12-2.57 \mathrm{~ms}\right)$ for the transient state (Supplementary Fig. 2 and Supplementary Table 2) that are similar to those reported previously for transient $H G$ bps $\left(p_{\mathrm{B}}=\sim 0.14-0.49 \% \text { and } \tau_{\mathrm{B}}=\sim 0.3-2.5 \mathrm{~ms}\right)^{1,2}$. The chemical shifts $\left(\omega_{\mathrm{B}}\right)$ of the transient state obtained using this analysis (Supplementary Fig. 3 and Supplementary Table 2) are also consistent with HG bps, including significantly downfield shifted purine $\mathrm{C} 8$ ( $\Delta \omega \approx 2.72$ p.p.m.), purine $\mathrm{Cl}^{\prime}(\Delta \omega \approx 3.41$ p.p.m.), cytosine C6 $(\Delta \omega \approx 2.40$ p.p.m. $)$ and upfield shifted imino $\mathrm{N} 1 / 3$ $(\Delta \omega \approx-1.84 \text { p.p.m. })^{1,2}$. Consistent with $H G$ bps, we did not observe significant chemical exchange at adenine $\mathrm{C} 2$ and thymine C6, which do not experience large chemical shift changes upon HG bp formation ${ }^{1,2}$ (Supplementary Fig. 1 and Supplementary Table 2). These results show that transient HG bps are not confined to CA/TG and TA/TA steps but rather occur broadly across a wide variety of sequence and positional contexts, including GA/TC, AA/TT, TA/TA, GG/CC, CG/CG and TG/ CA dinucleotide steps (where the $\mathrm{HG}$ bp is underlined).

Position- and sequence-dependent energetic variability. We observe $\sim 30$-fold variations in the transient HG population $(0.08$ $2.73 \%$ and $0.13-2.11 \%$ for $\mathrm{A} \bullet \mathrm{T}$ and $\mathrm{G} \bullet \mathrm{C}^{+}$bps, respectively) and $\sim 20$-fold variations in lifetimes $(0.12-2.57 \mathrm{~ms}$ and $0.40-2.08 \mathrm{~ms}$ for $\mathrm{A} \bullet \mathrm{T}$ and $\mathrm{G} \bullet \mathrm{C}^{+}$bps, respectively) (Supplementary Fig. 2 and Supplementary Table 2). This corresponds to $\sim 2.1 \mathrm{kcal} \mathrm{mol}^{-1}$ and $\sim 2.8 \mathrm{kcal} \mathrm{mol}^{-1}$ variations in the relative thermodynamic stability $\left(\Delta \Delta G_{\mathrm{WC}-\mathrm{HG}}\right.$ with $\left.\Delta G_{\mathrm{WC}-\mathrm{HG}}=G_{\mathrm{HG}}-G_{\mathrm{WC}}\right)$ (Fig. 2a) and forward free-energy barriers $\left(\Delta \Delta G^{\ddagger} \mathrm{WC}-\mathrm{HG}\right.$ with $\Delta G^{\ddagger} \mathrm{WC}-$ ${ }_{\mathrm{HG}}=G_{\mathrm{TS}}-G_{\mathrm{WC}}$, where $\mathrm{TS}$ is the transition state), respectively (Fig. 2b). These variations could reflect real sequence or position dependencies for transient HG bp formation. Alternatively, they could arise due to small differences in buffer conditions used for some of the duplexes (see Methods), particularly $\mathrm{pH}$, which can affect the energetics of transient $\mathrm{G} \bullet \mathrm{C}^{+} \mathrm{HG}$ bp formation ${ }^{1-3}$. However, systematic deviations in $\Delta G_{\mathrm{WC}-\mathrm{HG}}$ and $\Delta G^{\ddagger}$ WC-HG are not observed across DNA duplexes (Supplementary Fig. 4a). Furthermore, no correlations are observed between duplex melting temperatures measured by circular dichroism (CD) (Supplementary Fig. 4b,c and Supplementary Table 3) and either $\Delta G_{\mathrm{WC}-\mathrm{HG}}$ or $\Delta G^{\ddagger} \mathrm{WC}-\mathrm{HG}$ (Supplementary Fig. 4a). Consistent with sequence- and/or position-dependent contributions, the variations in $\Delta G_{\mathrm{WC}-\mathrm{HG}}$ and $\Delta G^{\ddagger}{ }_{\mathrm{WC}-\mathrm{HG}}$ are smaller $\left(\sim 1 \mathrm{kcal} \mathrm{mol}^{-1}\right)$ when comparing bps across different duplexes that share identical $5^{\prime}$ and $3^{\prime}$ neighbours and positions relative to duplex ends (indicated using horizontal lines in Fig. 2a,b; Supplementary Fig. 2 and Supplementary Table 2).

We observe significant variations in $\Delta G_{\mathrm{WC}-\mathrm{HG}}$ and $\Delta G^{\ddagger} \mathrm{WC}-\mathrm{HG}$ for the same dinucleotide step, which may arise due to differences in position and/or differences in the broader sequence context. 
a

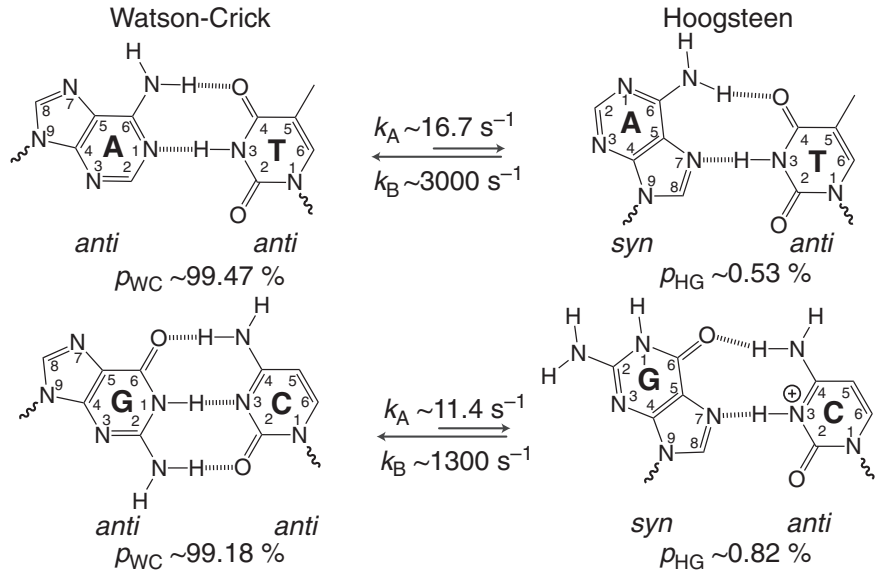

b

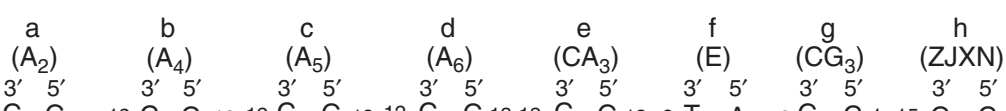
${ }_{2} \mathrm{C}-\mathrm{G}_{13} 12 \mathrm{C}-\mathrm{G}_{13} 12 \mathrm{C}-\mathrm{G}_{13} 12 \mathrm{C}-\mathrm{G}_{13} 12 \mathrm{C}-\mathrm{G}_{13} 8 \mathrm{P}-\mathrm{A}_{1} 6 \mathrm{G}-\mathrm{C}_{1} 15 \mathrm{G}-\mathrm{C}_{16}$ $11 G-C 1411 G-C 1411 G-C 1411 G-C 1411 \mathrm{G}-C_{14} 7 \mathrm{G}-\mathrm{C}_{2} \quad \mathrm{5}-\mathrm{C}-\mathrm{G} 214 \mathrm{C}-\mathrm{G} 17$ $10 G-C 1510 G-C 1510 G-C 1510 G-C 1510 G-C=15 \quad 6 \quad C-G_{3} \quad 4 G-C=13 \quad G-C 18$

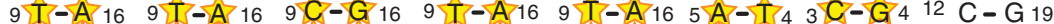

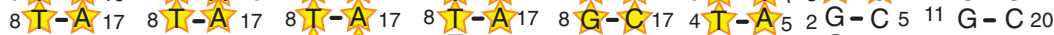

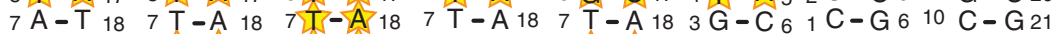
$6 \mathrm{G}-\mathrm{C}_{19} 6 \mathrm{6} \mathrm{T}-\mathrm{A}_{19} \quad 6 \mathrm{~T}-\mathrm{A}_{19} \quad 6 \mathrm{~T}-\mathrm{A} 19 \quad 6 \mathrm{G}-\mathrm{C}_{19}{ }_{2} \mathrm{C}-\mathrm{G}_{7} \quad 5^{\prime} \quad 3^{\prime} \quad 9 \mathrm{G}-\mathrm{C}_{22}$ $5 \mathrm{C}-\mathrm{G} 20 \quad 5 \mathrm{~A}-\mathrm{T} 20 \quad 5 \mathrm{~T}-\mathrm{A} 20 \quad 5 \mathrm{~T}-\mathrm{A} 20 \quad 5 \mathrm{~T}-\mathrm{A} 201 \mathrm{~A}-\mathrm{T} 8 \quad 8 \mathrm{G}-\mathrm{C} 23$

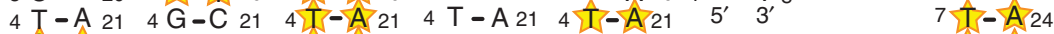
$3 \mathrm{~A}-\mathrm{T} 22$ 3 $\mathrm{C}-\mathrm{G} 22$ 3A-T 22 3 A - T22 3 A $\mathrm{A}-\mathrm{T} 22$ 2 C -G $23 \quad 2 \mathrm{G}-\mathrm{C} 23 \quad 2 \mathrm{G}-\mathrm{C} 23 \quad 2 \mathrm{G}-\mathrm{C} 23 \quad 2 \mathrm{G}-\mathrm{C} 23$ $1 G-C 24 \quad 1 C-G 24 \quad 1 C-G 24 \quad 1 C-G 24 \quad 1 C-G 24 \quad 4$ T - A 27

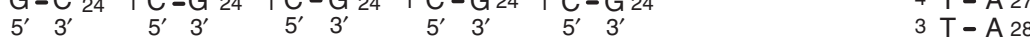
$2 \mathrm{G}-\mathrm{C} 29$ $1 \mathrm{G}-\underset{5^{\prime}}{-} \mathrm{C}^{\prime} \mathrm{C}$

C
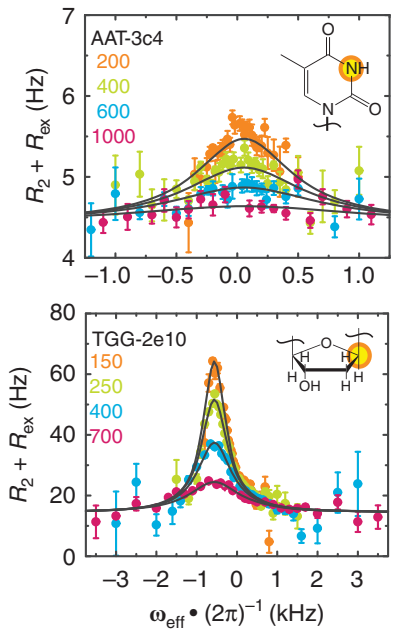
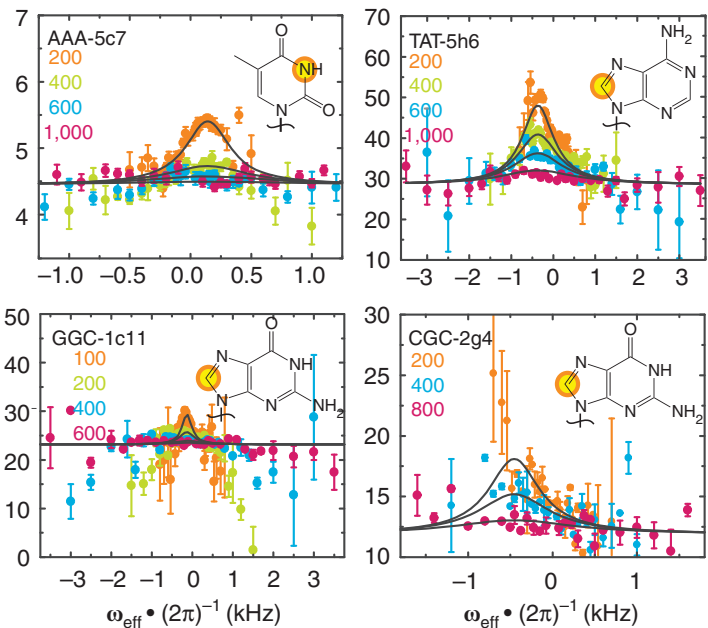

Figure 1 | Widespread occurrence of transient A•T and G•C ${ }^{+}$Hoogsteen base pairs in canonical duplex DNA. (a) The equilibrium between WatsonCrick and Hoogsteen base pairs. Shown are the average forward $\left(k_{A}\right)$ and reverse $\left(k_{B}\right)$ rate constants and populations of Watson-Crick $\left(p_{A}\right)$ and Hoogsteen $\left(p_{B}\right)$ base pairs obtained in this study. (b) DNA duplexes used in this study. Base pairs targeted for relaxation dispersion measurements are highlighted with a star. (c) Representative off-resonance ${ }^{13} \mathrm{C}$ and ${ }^{15} \mathrm{~N} R_{1}$ r relaxation dispersion profiles showing chemical exchange outside TA and CA steps of canonical duplex DNA. Spin lock powers $(\mathrm{Hz})$ are shown in the inset. Data are fit to equation 2. Error bars represent experimental uncertainty (one standard deviation) estimated from monoexponential fitting of duplicate $R_{1 \rho}$ data and analysis of signal-to-noise. See Methods for buffer conditions.

Notwithstanding these variations, the average stabilities of HG bps relative to $\mathrm{WC}$ bps $\left(\Delta \mathrm{G}_{\mathrm{WC}-\mathrm{HG}}\right)$ calculated for individual dinucleotide steps (Fig. 2a) follow an order (TA/TA $>\mathrm{AA} /$ $\mathrm{TT}>\mathrm{CA} / \mathrm{TG}>\mathrm{GA} / \mathrm{TC}$ for $\mathrm{A} \bullet \mathrm{T}$ and $\mathrm{TG} / \mathrm{CA} \geq \mathrm{CG} / \overline{\mathrm{CG}} \geq \mathrm{GG} / \overline{\mathrm{CC}}$ for $\mathrm{G} \bullet \overline{\mathrm{C}}^{+}$) that is nearly inverted relative to the well-documented WC dinucleotide stabilities (GA/TC $\geq \mathrm{CA} / \mathrm{TG}>\mathrm{AA} / \mathrm{TT}>\mathrm{TA} / \mathrm{TA}$ for $\mathrm{A} \bullet \mathrm{T}$ and $\mathrm{CG} / \mathrm{CG}>\mathrm{GG} / \mathrm{CC}>\mathrm{TG} / \mathrm{CA}$ for $\mathrm{G} \bullet \mathrm{C})$, which measure the stability of WC bps relative to the melted state ${ }^{31}$. Thus, transient HG bps seem to be more abundant in less stable
WC dinucleotide steps such as CA/TG and TA/TA steps. The energetic preference for HG bps at TA/TA steps observed here is consistent with a large body of data showing that HG bps are favoured in A-T-rich sequences, particularly TA/TA steps (reviewed in ref. 8).

Origin of variable transient HG bp energetics. Strikingly, we observe a clear correlation $(R=0.76)$ between $\Delta G_{\mathrm{WC}-\mathrm{HG}}$ and 

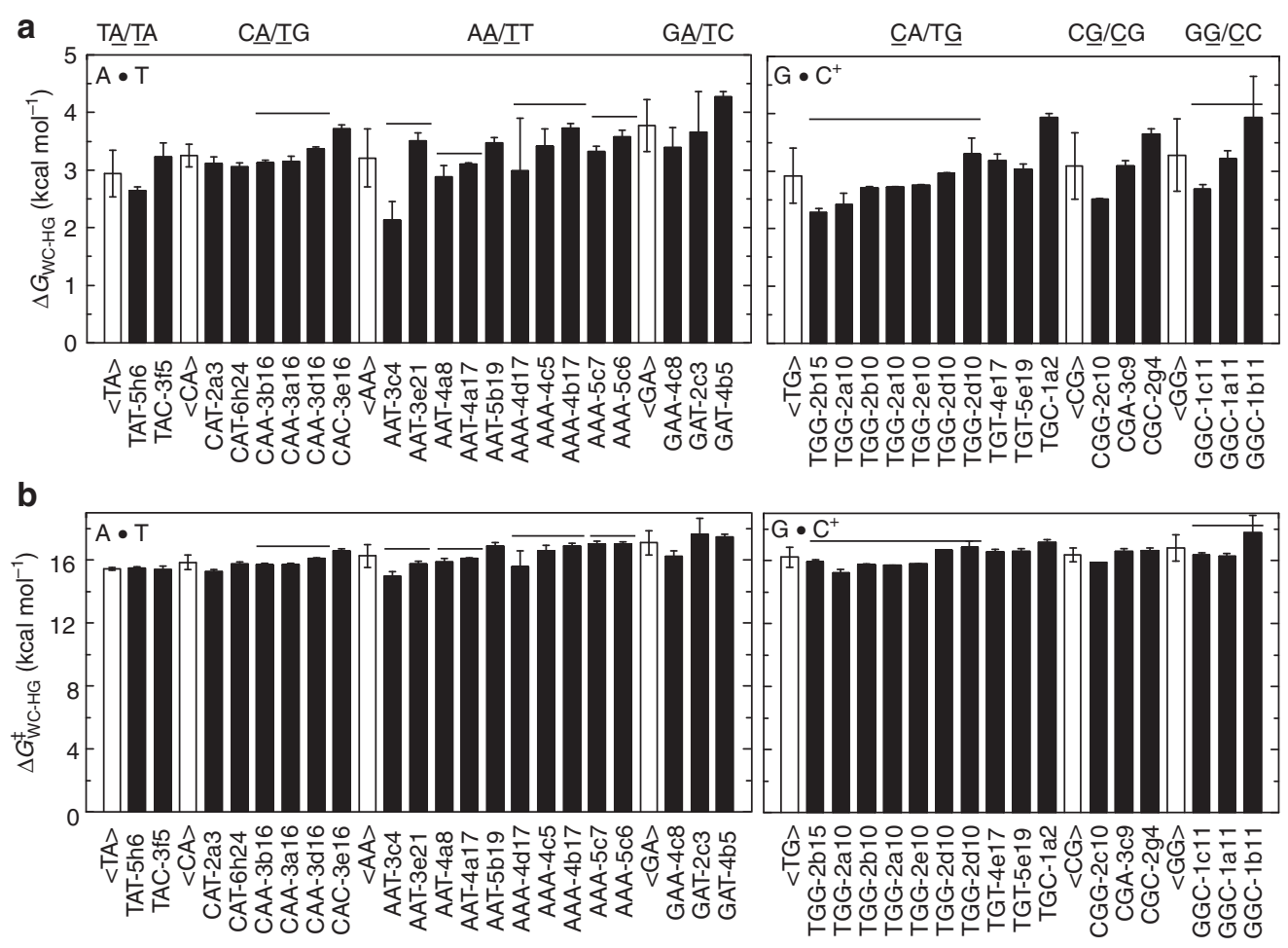

C

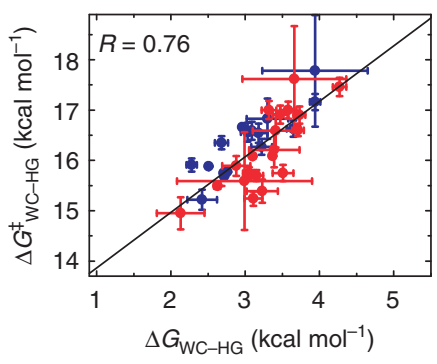

d

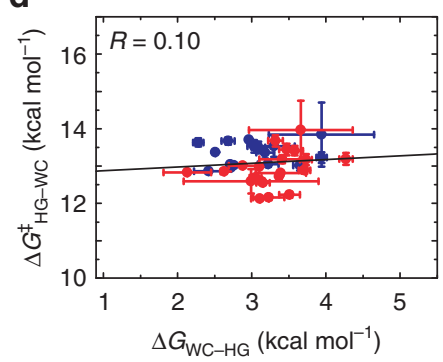

e

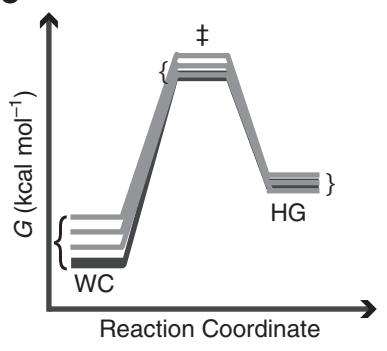

Figure 2 | Sequence- and position-dependent thermodynamic and kinetic parameters describing the Watson-Crick to Hoogsteen transition. (a) Freeenergy difference $\left(\Delta G_{W C}-H G\right)$ and (b) forward free-energy barrier $\left(\Delta G^{\ddagger} W C-H G\right)$ for the Watson-Crick to Hoogsteen transition as derived from a two-state analysis of the $R_{1 \rho}$ data. Error bars represent experimental uncertainty (one s.d.) estimated from propagation of errors from monoexponential fitting of duplicate $R_{1 \rho}$ data and analysis of signal-to-noise. Average and standard deviations for dinucleotide steps are shown using white bars. Horizontal lines denote bps with same triplet sequence and positions relative to nearest terminal end. $X$ axis labels denote the triple sequence context (' $X Y Z$ ') with Hoogsteen base pair in the middle; the position relative to the closest terminal end $\left({ }^{\prime}-n^{\prime}\right)$, and name of duplex as denoted in Fig. $1 b$. Correlation between (c) $\Delta G^{\ddagger} W C-H G$ and $\Delta G_{W C}-H G$ as well as (d) $\Delta G^{\ddagger} H G-W C$ and $\Delta G_{W C}-H G$. A $\bullet T$ and $G \bullet C+$ base pairs are shown in red and blue, respectively. The best line of fit and corresponding Pearson coefficient $(R)$ are shown. (e) Free-energy diagram of the Watson-Crick to Hoogsteen transition depicting relative variations in free energy of Watson-Crick, transition and Hoogsteen states.

$\Delta G^{\ddagger}$ WC-HG (Fig. 2c) and a relatively uniform backward freeenergy barrier $\left(\Delta G^{\ddagger} \mathrm{HG}-\mathrm{wC}=G_{\mathrm{TS}}-G_{\mathrm{HG}}\right)$ of $\sim 13 \mathrm{kcal} \mathrm{mol}^{-1}$ (Fig. 2d). Thus, changing the sequence context has little effect on the relative energetic stability of the TS and HG bp. This could either be because the stabilities of the TS and HG bp are not significantly affected by changes in sequence, or because their stabilities vary in a correlated manner. In contrast, a change in sequence context does change the relative stability of both the TS and $\mathrm{HG}$ bp relative to the WC bp (Fig. 2e).

One possibility is that sequence-specific variations are dominated by changes in variations in the WC bp without significantly affecting the stabilities of the TS and HG bp (Fig. 2e). Indeed, the observed variations in $\mathrm{HG}$ bp stability $\left(\sim 2.1 \mathrm{kcal} \mathrm{mol}^{-1}\right)$ are comparable in size with variations in WC bp stability $\left(\sim 2 \mathrm{kcal} \mathrm{mol}^{-1}\right)$ measured across dinucleotide steps using melting experiments ${ }^{31}$. This would also explain why transient $\mathrm{HG}$ bps are specifically more abundant at dinucleotide steps that have weakened WC stabilities (Fig. 2a,b). If the observed variations are indeed dominated by variations in WC stability, one might expect a similar correlation between the $\Delta G_{\mathrm{cl}-\text { op }}$ and $\Delta G_{\text {cl-op }}^{\ddagger}$ values describing transitions between WC bps and the bp open state, especially since stability of the open state is not expected to vary significantly with sequence. Indeed, a previous analysis of $\Delta G_{\mathrm{cl}-\mathrm{op}}$ and $\Delta G_{\mathrm{cl}-\text { op }}^{\ddagger}$ correlation reported by Coman and Russu $^{32}$ based on imino proton exchange measurements ${ }^{33}$ reveals a comparably strong correlation $(R=0.8)^{34}$. Our results suggest that the free energy of the TS varies less relative to the HG bp with sequence/position as compared with the WC bp. If one were to assume that a similar sequence/ position-dependent free energy implies a similar structure, even if the sequence/position dependence is very small, then these results would suggest that the TS is structurally more similar to the HG bp-consistent with a 'late' TS. 
$\Phi$-value analysis suggests a 'late' transition state. To quantify the extent to which the sequence-specific TS energetics are more similar to HG bps versus WC bps, we subjected the measured $\Delta \mathrm{G}_{\mathrm{WC}-\mathrm{HG}}$ and $\Delta G^{\ddagger} \mathrm{WC}-\mathrm{HG}$ values to $\Phi$-value analysis ${ }^{34,35}$ (Methods and Fig. 3). In this approach, one computes a $\Phi$ value, which quantifies the relative magnitude of the sequence/ position-dependent free-energy differences between the TS and WC bps and those between WC bps and HG bps,

$$
\Phi=\Delta \Delta G_{\mathrm{TS}-\mathrm{WC}} / \Delta \Delta G_{\mathrm{WC}-\mathrm{HG}}
$$

where $\Delta \Delta G_{\mathrm{TS}}-\mathrm{WC}=\left(G_{\mathrm{TS}}-G_{\mathrm{WC}}\right)_{\mathrm{mut}}-\left(G_{\mathrm{TS}}-G_{\mathrm{WC}}\right)_{\Psi-\mathrm{WT}}$ and $\Delta \Delta G_{\mathrm{WC}-\mathrm{HG}}=\left(G_{\mathrm{HG}}-G_{\mathrm{WC}}\right)_{\mathrm{mut}}-\left(G_{\mathrm{HG}}-G_{\mathrm{WC}}\right)_{\Psi-\mathrm{WT}}$ are the changes in the forward free-energy barrier and free-energy difference between WC and HG bps, respectively, on mutating (mut) the sequence/position of a reference $(\Psi-\mathrm{WT}) \mathrm{bp}$. It is instructive to consider two limiting cases to help understand how this analysis can be used to quantify the extent to which the sequence-specific TS energetics are more similar to HG bps versus WC bps and whether a TS is 'early' or 'late'. In the case that the TS and HG share identical sequence-specific energetics as might be expected for a late TS, a given sequence-specific perturbation equally affects $G_{\mathrm{TS}}$ and $\mathrm{G}_{\mathrm{HG}}$ (that is, $G_{\mathrm{HG}}-G_{\mathrm{TS}}=$ constant for all mutants) and $\Delta \Delta G_{\mathrm{TS}-\mathrm{WC}}=\Delta \Delta G_{\mathrm{WC}-\mathrm{HG}}$ and $\Phi=1$. On the other hand, if TS and WC share identical sequence-specific energetics as might be expected for an early TS, then $G_{\mathrm{TS}}-G_{\mathrm{WC}}=$ constant for all mutants and $\Delta \Delta G_{\mathrm{TS}-\mathrm{WC}}=0$ and $\Phi=0$. In practice, the $\Phi$-value can range between 0 and 1 , with intermediate $\Phi$-values being more difficult to interpret in the context of a structural mechanism $^{14}$.

We arbitrarily assign reference $\mathrm{A} \bullet \mathrm{T}$ and $\mathrm{G} \bullet \mathrm{C}$ bps to be those having the smallest $\Delta G_{\mathrm{WC}-\mathrm{HG}}$ values, and which therefore form the most stable HG bps among those studied herein $(\Psi-\mathrm{WT}$, Fig. 3a,b). Next, we computed $\Phi$ for each $\mathrm{A} \bullet \mathrm{T}$ and $\mathrm{G} \bullet \mathrm{C}^{+} \mathrm{bp}$. This analysis was preformed separately for $\mathrm{A} \bullet \mathrm{T}$ and $\mathrm{G} \bullet \mathrm{C}^{+}$bps and repeated multiple times assuming a different bp as the designated wild-type reference (data not shown). In the vast majority of the cases, we measure $\Phi$-values near 1 , consistent with a 'late' TS (Fig. 3c and Supplementary Table 4). It should be noted that similar sequence/position-dependent energetics for the TS and HG bp does not have to imply similarity in structure, and that we cannot rule out the possibility of an early TS that has structural features similar to WC but sequence/position-dependent energetics that are more similar to HG.

\section{Discussion}

Our results suggest that HG bps can occur ubiquitously in canonical duplex DNA across different sequence and positional contexts. This can help explain how polymerases such as the human DNA polymerase iota can use HG pairing as a general mechanism to replicate DNA and thereby bypass lesions that diminish the ability to form WC bps ${ }^{8,16}$. Our findings also raise the possibility that HG bps may be widespread in genomic DNA, especially given that the energetic differences between $\mathrm{WC}$ and a

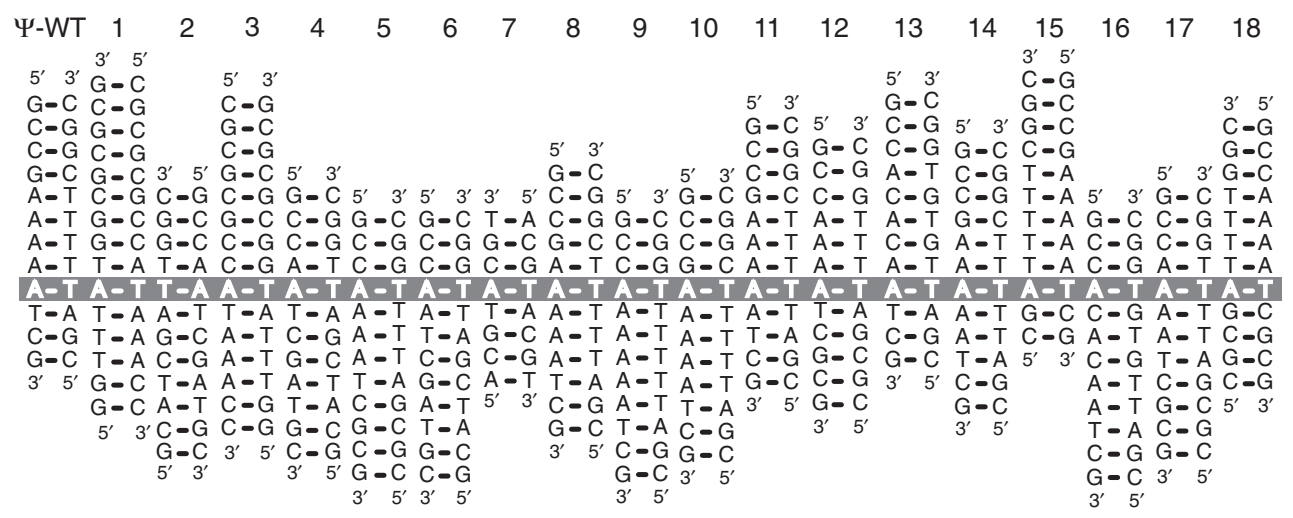

b

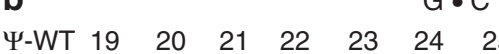

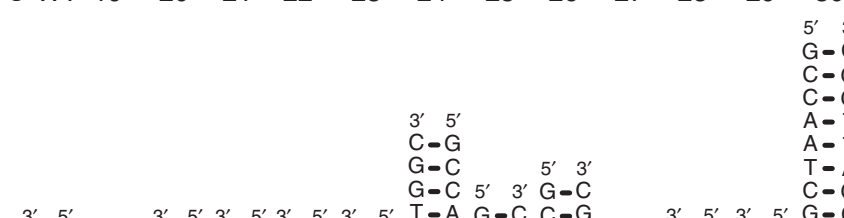

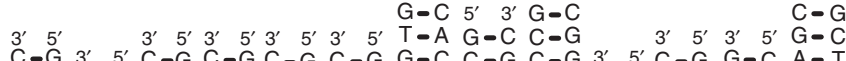

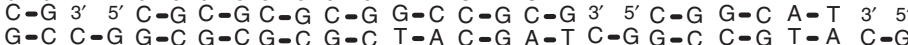

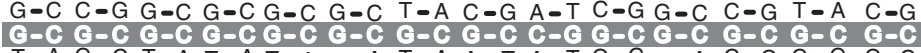

$T-A$ G-C T-A T-A T-A T-A T-A A-T A-T G-C T-A C-G C-G G-C

T-A C-G T-A T-A G-C T-A T-A A-T C-G T-A T-A G-C $3^{\prime} 5^{\prime} T-A$

$A-T$ T-A T-A A-T T-A T-A A-T A-T A-T T-A T-A C- $G$

$G-C$ T-A T-A G-C G-C T-A G-C A-T A-T A-T T-A $5^{\prime} 3^{\prime}$

$C-G T-A$ A-T C-G T-A T-A C-G A-T T-A G-C T-A

$T-A T-A$ G-C T-A T-A T-A $5^{\prime} 3^{\prime} T-A$ C-G C-G T-A

$A-T$ T-A C-G A-T A-T A-T

A-G $A-T$ G $-C$ C $-G A-C$ G $-T$

A-G $A-T, G-C$ C $-G G-C G-C$

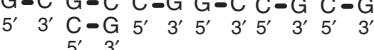

$C-G G-C$ T $-A$ A - T

$G-C 3^{\prime} 5^{\prime} A-T G-C$

$3^{\prime} 5^{\prime} \quad C-G C-G$

$C-G$
$G-C$
$5^{\prime}$
C

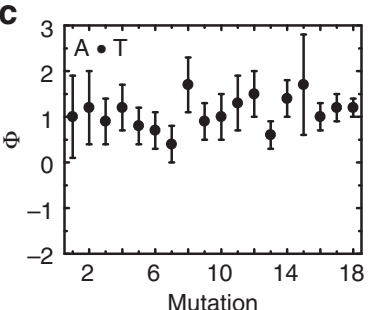

C

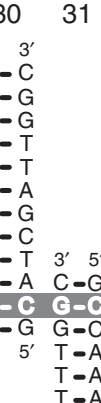

$T=A$
$T=A$
$T=A$

$T=A$
$T=A$
$A-T$

$\mathrm{G}-\mathrm{C}$
$\mathrm{C}-\mathrm{G}$

C-G

G-C

$C-G$
$5^{\prime} \quad 3^{\prime}$

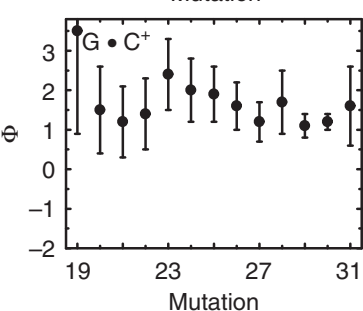

Figure 3 | $\mathbf{\Phi}$-value analysis. Perturbing the Watson-Crick to Hoogsteen equilibrium in (a) A $\bullet T$ and (b) $\mathrm{G}_{\bullet} \mathrm{C}^{+}$base pairs by varying the sequence and/or positional context of individual base pairs. All duplexes are re-drawn after aligning each $\mathrm{A} \bullet T$ or $\mathrm{G} \bullet \mathrm{C}^{+}$base pair with a reference sequence variant $(\Psi-W T)$. (c) $\Phi$-values for $A \bullet T$ and $G \bullet C^{+}$base pairs. The sequence variant number is shown above each secondary structure. 
HG bps are small compared with forces in living cells, including those arising due to supercoiling, torsional stress, and protein binding. It is worth noting that difficulties in distinguishing between WC and HG bps based on X-ray crystallography data have been reported ${ }^{8,36}$. Our finding that transient HG bps can occur ubiquitously in duplex DNA calls for the re-examination of current X-ray structures of DNA to more critically assess for the occurrence of HG bps.

Our studies suggest that the occurrence of HG bps depends in a complex manner on both sequence context and position. This is consistent with the hypothesis by Honig and Rohs ${ }^{37}$ that the observation of HG base pairing makes it is unlikely that proteinDNA binding is driven by a simple linear code. Nevertheless, our results together with prior studies ${ }^{8}$ suggest that HG bps are likely to exist in greater abundance within unstable and structurally stressed environments, such as kinks and turns, which can destabilize WC bps, including stacking interactions. Indeed, while only a few X-ray structures have documented the existence of HG bps in DNA, in many cases HG bps occur near structurally stressed environments. For example, HG bps are observed near kinks or nicks in X-ray structures of DNA bound to TATA boxbinding protein ${ }^{11}$ and integration host factor ${ }^{9}$, and near a hairpin loop in structures of DNA bound to TnpA transposase ${ }^{38}$. HG bps have also been observed for DNA in complex with antibiotics that contribute unique stacking interactions ${ }^{27,39}$. Previous studies ${ }^{3}$ have shown that changes in counterion concentration have a measurable effect on the population and lifetime of HG bps. Future studies should therefore also examine how the sequenceand position-dependent $\mathrm{HG}$ energetics vary with increasing counterion concentration $\left(\mathrm{Na}^{+}\right.$and $\left.\mathrm{Mg}^{2+}\right)$. Studies so far suggest that $\mathrm{Na}^{+}$and $\mathrm{Mg}^{2+}$ stabilize A•T HG but destabilize $\mathrm{G} \bullet \mathrm{C}^{+} \mathrm{HG}$ bps in the case of CA/TG steps ${ }^{3}$. Although further studies are needed to more quantitatively understand the sequence and position dependence of transient HG bp formation, these energetic preferences may provide a new mechanism for shape-based DNA recognition ${ }^{12,19}$ via indirect read-out mechanisms ${ }^{40}$. Our study has focused on the occurrence of single transient HG bps surrounded by WC bps. Additional studies are needed to examine sequence-specific propensities for forming longer stretches of HG bps, and HG tracts interspersed by WC bps. Such mixtures of WC and HG bps can endow genomic DNA with a new level of structural complexity similar to Z-DNA.

Our results suggest that the sequence- and position-specific variations in $\mathrm{HG}$ bp stabilities and lifetimes are dominated by variations in the WC bp stability and to a lesser extent by variations in the stabilities of the TS and HG bp. Interestingly, a similar trend has been reported for base opening ${ }^{32}$. Future studies should further explore the WC-to-HG transition pathways and examine whether they share a similar TS with base opening and whether there can be pathways toward HG that proceed via the base-opened state. Conjugate peak refinement simulations suggest a pathway in which the purine base rotates toward the major groove inside the double helix ${ }^{1}$; however, further experimental characterization is required. The observation that the sequence and/or position variations in the TS free energies are more similar to the HG bp versus the WC bp suggests the TS is structurally more similar to HG versus WC, consistent with a 'late' TS for the WC-to-HG transition. However, we cannot rule out an early TS that is structurally more similar to WC but has sequence/ position-dependent energetics that are more similar to the HG bp. Although the structure of the TS remains unclear, an equally important question is why the energetic stabilities of $\mathrm{HG}$ bps appear to be only weakly dependent on sequence. Further studies are required to understand the structure and specific interactions that may help stabilize the TS and HG bp.

\section{Methods}

NMR samples and resonance assignments. Unlabelled DNA samples were purchased as single-stranded oligos from Integrated DNA Technologies (IDT) with standard desalting purification. The DNA oligos were resuspended to $\sim 200 \mu \mathrm{M}$ in $15 \mathrm{mM}$ phosphate buffer with corresponding $\mathrm{pH}$ (see below), $25 \mathrm{mM} \mathrm{NaCl}, 0.1 \mathrm{mM}$ EDTA. Duplexes were annealed by mixing an equimolar ratio of the complementary DNA strands, heating at $95^{\circ} \mathrm{C}$ for 2 min followed by gradual cooling at room temperature for $\sim 30 \mathrm{~min}$. Unlabelled DNA duplexes were washed $3 \times$ in resuspension buffer by microcentrifugation using an Amicon Ultra- 4 centrifugal filter with a $3-\mathrm{kDa}$ cutoff, concentrated to $\sim 2-3 \mathrm{mM}$ and $\sim 250 \mu \mathrm{l}$, then supplied with $10 \% \mathrm{D}_{2} \mathrm{O}$. Natural abundance $\mathrm{CG}_{3}$ was resuspended in $\sim 4 \mathrm{ml}$ of milliQ $\mathrm{H}_{2} \mathrm{O}$ and dialyzed against $2 \mathrm{l}$ of milliQ $\mathrm{H}_{2} \mathrm{O}$ with two exchanges for a total of 61 of milliQ $\mathrm{H}_{2} \mathrm{O}$, using a dialysis tube from $\mathrm{G}$-Biosciences with a 1-kDa cutoff. Dialyzed $\mathrm{CG}_{3}$ was lyophilized and resuspended in NMR buffer to $\sim 4 \mathrm{mM}$ and supplied with $10 \% \mathrm{D}_{2} \mathrm{O}$. Hemi- ${ }^{13} \mathrm{C}^{15} \mathrm{~N}$-labelled $\mathrm{A}_{5}$ duplex was prepared by annealing a uniformly ${ }^{13} \mathrm{C} /{ }^{15} \mathrm{~N}$-labelled thymine-rich strand to a natural abundance adenosinerich strand. Fully ${ }^{13} \mathrm{C} /{ }^{15} \mathrm{~N}$-labelled DNA duplexes were prepared by annealing two labelled strands together. All labelled single strands were synthesized in vitro by the method of Zimmer and Crothers ${ }^{41}$ using a DNA hairpin template with a $5^{\prime}$ overhang corresponding to the complement of the target labelled strand and a $3^{\prime}$ ribose (IDT). In this study, we used the same hairpin sequence as Zimmer and Crothers $^{41}$, Klenow fragment DNA polymerase (NEB) NEB2 buffer (NEB) and uniformly ${ }^{13} \mathrm{C} /{ }^{15} \mathrm{~N}$-labelled dNTPs (Isotec, Sigma-Aldrich and Silantes). Base- and heat-catalyzed cleavage separated the hairpin template from the ${ }^{13} \mathrm{C} /{ }^{15} \mathrm{~N}$-labelled synthesized product. The single-stranded DNA product was purified by $20 \%$ denaturing polyacrylamide gel electrophoresis, isolated by passive elution from crushed gel pieces and desalted on a C18 reverse-phase column (Sep-Pak, Waters). The oligo was lyophilized and suspended in NMR buffer. The semi-labelled DNA samples were prepared by titrating the unlabelled strand directly into an NMR tube containing the ${ }^{13} \mathrm{C} /{ }^{15} \mathrm{~N}$-labelled strand and monitoring the disappearance of single-stranded DNA peaks using heteronuclear single quantum coherence (HSQC) experiments. The fully labelled samples were annealed in a similar fashion. $2 \mathrm{D}{ }^{1} \mathrm{H}-{ }^{1} \mathrm{H}$ nuclear overhauser spectroscopy (NOESY) experiments at $26{ }^{\circ} \mathrm{C}\left(\mathrm{A}_{2}\right.$, $\left.\mathrm{A}_{4}, \mathrm{~A}_{5}, \mathrm{~A}_{6}, \mathrm{CA}_{3}, \mathrm{E}\right)$ or $25^{\circ} \mathrm{C}\left(\mathrm{CG}_{3}\right.$ and $\mathrm{Z}$ junction (ZJXN)) and $\mathrm{pH} 5.2\left(\mathrm{~A}_{5}\right), 5.4\left(\mathrm{~A}_{2}\right.$, $\left.\mathrm{A}_{4}, \mathrm{~A}_{6}, \mathrm{CA}_{3}, \mathrm{CG}_{3}\right), 6.8(\mathrm{E})$ or $7.5(\mathrm{ZJXN})$ were used to assign resonances as described previously ${ }^{1}$. See NMR $R_{1 \rho}$ relaxation dispersion for $R_{1 \rho} \mathrm{RD} \mathrm{pH}$ values.

NMR $\boldsymbol{R}_{\mathbf{1} \rho}$ RD. All NMR experiments were performed on a Bruker Avance $600 \mathrm{MHz}$ NMR spectrometer equipped with a $5-\mathrm{mm}$ triple-resonance cryogenic probe. $R_{1 \rho} \mathrm{RD}$ experiments were performed at $\mathrm{pH} 5.2-7.5$ and 5.2-5.4 for $\mathrm{A} \bullet \mathrm{T}$ and $\mathrm{G} \bullet \mathrm{C}^{+}$bps, respectively. Buffer conditions for $R_{1 \rho} \mathrm{RD}$ measurements are $15 \mathrm{mM}$ sodium phosphate, $25 \mathrm{mM} \mathrm{NaCl}, 0.1 \mathrm{mM}$ EDTA, $10 \% \mathrm{D}_{2} \mathrm{O} \mathrm{pH}=5.2\left(\mathrm{~A}_{5}, \mathrm{~A}_{4}\right.$ : $\mathrm{C} 15$ C6, A16 C1', $\left.\mathrm{G} 10 \mathrm{Cl}^{\prime}\right), \mathrm{pH}=5.4\left(\mathrm{~A}_{2}, \mathrm{~A}_{4}, \mathrm{~A}_{6}, \mathrm{CA}_{3}, \mathrm{CG}_{3}\right), \mathrm{pH}=6.8(\mathrm{E})$ and $\mathrm{pH}=7.5(\mathrm{ZJXN})$ at $26^{\circ} \mathrm{C}\left(\mathrm{A}_{2}, \mathrm{~A}_{4}, \mathrm{~A}_{5}, \mathrm{~A}_{6}, \mathrm{CA}_{3}, \mathrm{E}\right)$ or $25^{\circ} \mathrm{C}\left(\mathrm{A}_{2} \mathrm{~A} 3 \mathrm{Cl}^{\prime}, \mathrm{CG}_{3}\right.$, ZJXN). $C_{3}$ and $E$ are unlabelled DNA samples, the T-rich strand of $A_{5}$ is ${ }^{13} \mathrm{C} /{ }^{15} \mathrm{~N}$ labelled while $\mathrm{A}_{6}, \mathrm{~A}_{4}, \mathrm{~A}_{2}, \mathrm{CA}_{3}$ and ZJXN are fully ${ }^{13} \mathrm{C} /{ }^{15} \mathrm{~N}$-labelled. We use low-pH conditions for transient (and protonated) $\mathrm{G} \bullet \mathrm{C}^{+}$bps in order to increase the WCto-HG chemical exchange signature detected by $R_{1 \rho} \mathrm{RD}^{3}$. We recently reported a detailed analysis of the $\mathrm{pH}$ dependence of chemical exchange corresponding to transient $\mathrm{HG}$ bp formation and how measurements at such lower $\mathrm{pH}$ conditions can be qualitatively interpolated to assess exchange at higher $\mathrm{pH}^{3}$. Carbon and nitrogen $R_{1 \rho} \mathrm{RD}$ profiles for guanine/adenine $\mathrm{C} 8$, guanine/adenine $\mathrm{C1}^{\prime}$, cytosine C6, guanine $\mathrm{N} 1$ and thymine $\mathrm{N} 3$ were measured using a one-dimensional acquisition scheme which uses selective Hartmann-Hahn polarization transfer $^{42}$ to selectively excite one $\mathrm{C}-\mathrm{H}$ or $\mathrm{N}-\mathrm{H}$ spin system at a time ${ }^{6}$. The spin lock power and offset frequencies are summarized in Supplementary Table 4. The following delays were used: $\mathrm{A}_{2}$ : $\mathrm{A} 3 \mathrm{Cl}^{\prime}, \mathrm{G} 10 \mathrm{Cl}^{\prime}, \mathrm{A} 17 \mathrm{Cl}^{\prime}$; $\mathrm{A}_{4}$ : $\mathrm{A} 5 \mathrm{Cl}^{\prime}, \mathrm{G} 10 \mathrm{Cl}^{\prime}, \mathrm{A} 16 \mathrm{Cl}^{\prime}, \mathrm{A} 19 \mathrm{Cl}^{\prime}$; $\mathrm{CA}_{3}$ : $\mathrm{G}_{10 ~ \mathrm{Cl}^{\prime}}$; $\mathrm{A}_{5}$ : A3 C1', C5 C1 ${ }^{\prime}(0,4,8,12,18,26,34,42,12,42)$; $\mathrm{A}_{5} \mathrm{G} 11 \mathrm{C} 8(0$ $12,32,26,32) ; A_{4}$ A17 C8, A $A_{6}$ A17 C8 $(0,4,12,32,26,32) ; A_{2}$ : C2 C6, T9 C6, G11 C8, A16 C8, A17 C2; CA 3 : A16 C8, C17 C6, C19 C6, A21 C1'; A : C15 C6, G11 C8; $\mathrm{A}_{5}$ : C9 C6, G10 C8; A 6 : G10 C8, A16 C8 $(0,4,8,12,16,20,26,32,12,32) ; A_{2}$ : T8 N3, G10 N1, G23 N1; A 5 : T4 N3, T5 N3, T6 N3, T7 N3, T8 N3; A G10 N1 (0, 8, $16,24,36,48,60,80,100,16,70,100)$; $\mathrm{CG}_{3} \mathrm{G} 4 \mathrm{C} 8(0,60,60)$; ZJXN: A6 C8, A24 C8 $(0,4,8,12,16,20,24,30,12,30)$; E A5 C8: $(0,48,48)$. Data points meeting C-C Hartmann-Hahn matching conditions were omitted as described previously ${ }^{6}$. Data were processed using NMRPipe ${ }^{43}$ and $R_{1 \rho}$ values were determined from monoexponential decay fits of the resonance intensities using a script ${ }^{44}$ in Mathematica 9 (Wolfram Research). On- and off-resonance $R_{1 \rho}$ data were fit to the Laguerre equation $^{45}$ (equation 2) using Origin 8.6 (OriginLab),

$$
R_{1 \rho}=R_{1} \cos ^{2} \theta+R_{2} \sin ^{2} \theta+\frac{\sin ^{2} \theta p_{\mathrm{A}} p_{\mathrm{B}} \Delta \omega^{2} k_{\mathrm{ex}}}{\omega_{\mathrm{A}}^{2} \omega_{\mathrm{B}}^{2} / \omega_{\mathrm{eff}}^{2}+k_{\mathrm{ex}}^{2}-\sin ^{2} \theta p_{\mathrm{A}} p_{\mathrm{B}} \Delta \omega^{2}\left(1+\frac{2 k_{\mathrm{ex}}^{2}\left(p_{\mathrm{A}} \omega_{\mathrm{A}}^{2}+p_{\mathrm{B}} \omega_{\mathrm{B}}^{2}\right)}{\omega_{\mathrm{A}}^{2} \omega_{\mathrm{B}}^{2}+\omega_{\mathrm{eff}}^{2}}\right)}
$$

Where $\omega_{\text {eff }}^{2}=\Omega^{2}+\omega_{1}^{2}, \omega_{\mathrm{A}}^{2}=\left(\Omega_{\mathrm{A}}-\omega_{\mathrm{rf}}\right)^{2}+\omega_{1}^{2}$ and $\omega_{\mathrm{B}}^{2}=\left(\Omega_{\mathrm{B}}-\omega_{\mathrm{rf}}\right)^{2}+\omega_{1}^{2}$. $\Delta \omega_{\mathrm{AB}}=\Omega_{\mathrm{B}}-\Omega_{\mathrm{A}}$, where $\Omega_{\mathrm{A}}$ and $\Omega_{\mathrm{B}}$ are the chemical shifts of the ground (A) and transient (B) states, respectively, in Hz. $R_{1}$ and $R_{2}$ are the intrinsic longitudinal and transverse relaxation rate constants, respectively, and are assumed to be identical for the ground (A) and transient (B) states. $\theta=\arctan \left(\omega_{1} / \Omega\right)$ where $\omega_{1}$ is the spin lock power strength. $\Omega=\Omega_{\text {obs }}-\omega_{\text {rf }}$ where $\Omega$ is the offset of the spin lock carrier 
frequency $\left(\omega_{\mathrm{rf}}\right)$ from the observed resonance frequency $\left(\Omega_{\mathrm{obs}}\right)$. $\Omega_{\mathrm{obs}}=p_{\mathrm{A}} \Omega_{\mathrm{A}}+p_{\mathrm{B}} \Omega_{\mathrm{B}}$ where $p_{\mathrm{A}}$ and $p_{\mathrm{B}}$ are the ground and transient state populations, respectively, and $p_{\mathrm{A}}+p_{\mathrm{B}}=1 . k_{\mathrm{ex}}$ is the chemical exchange rate constant for a two-state exchange process where $k_{\mathrm{ex}}=k_{\mathrm{A}}+k_{\mathrm{B}}, k_{\mathrm{A}}=k_{\mathrm{ex}} p_{\mathrm{B}}$ and $k_{\mathrm{B}}=k_{\mathrm{ex}} p_{\mathrm{A}} \cdot k_{\mathrm{A}}$ and $k_{\mathrm{B}}$ are the forward and reverse rate constants, respectively.

Plots of $R_{1 \rho}$ data are presented as $R_{2 \text { eff }}\left(R_{2 \text { eff }}=R_{2}+R_{\text {ex }}\right)$ in Fig. 1c and Supplementary Fig. 1 where,

$$
R_{2, \text { eff }}=\frac{R_{1 \rho}}{\sin ^{2} \theta}-\frac{R_{1}}{\tan ^{2} \theta}
$$

In cases where large errors were accompanied by small $R_{\mathrm{ex}}$, model selection comparing presence and absence of exchange was carried out using the $\mathrm{F}$ - and Akaike's Information Criterion (AIC) ${ }^{46}$ tests to discriminate between exchange and no detectable exchange (data not shown). The F-test compares two nested models fit to the same data under the null hypothesis that the residual sum of squares of the less complex, restricted model is not significantly larger than that of the more complex, full model. The AIC test assesses the likelihood of a model given the data and seeks to minimize loss of information embedded within the data. For $A_{2}$ G23 $\mathrm{N} 1$, no exchange is the selected model under the conditions used. Because of lower sensitivity to exchange, we did not interpret absence of ${ }^{15} \mathrm{~N}$ dispersion as evidence for absence of transient HG bps.

The free-energy difference between the WC GS and HG transient state $\left(\Delta G_{\mathrm{WC}-\mathrm{HG}}\right)$ was computed using

$$
\Delta G_{\mathrm{WC}-\mathrm{HG}}=-R T\left(\ln \left(\frac{k_{1} h}{k_{\mathrm{B}} T}\right)-\ln \left(\frac{k_{2} h}{k_{\mathrm{B}} T}\right)\right)
$$

where $k_{1}$ and $k_{2}$ are the forward and reverse rate constants, respectively, $h$ is Planck's constant, $k_{\mathrm{B}}$ is Boltzmann's constant, $R$ is the gas constant and $T$ is temperature. The forward barrier of the transition $\left(\Delta G^{\ddagger} \mathrm{WC}-\mathrm{HG}\right)$ was computed using,

$$
\Delta G_{\mathrm{WC}-\mathrm{HG}}^{ \pm}=-R T \ln \left(\frac{k_{1} h}{\kappa k_{\mathrm{B}} T}\right)
$$

where $\kappa$ is the transmission coefficient which is assumed to be unity.

CD melting. DNA duplexes (IDT) were prepared using ultracentrifugation as described in NMR Samples and Resonance Assignments in $15 \mathrm{mM}$ phosphate buffer, $0.1 \mathrm{mM}$ EDTA, $25 \mathrm{mM} \mathrm{NaCl} \mathrm{pH} 5.4$ and supplied with $10 \% \mathrm{D}_{2} \mathrm{O}$. Duplexes were prepared by diluting complementary single-stranded stocks to $50 \mu \mathrm{M}$ in the same tube with a final volume of $200 \mu$ l. Samples were denatured at $95^{\circ} \mathrm{C}$ for $5 \mathrm{~min}$ followed by annealing of at least $10 \mathrm{~min}$ on the bench top. Samples were transferred to a 1-mm cuvette (Starna Cells), mineral oil was added to the top of the solution and the cuvette was capped. Melting experiments were performed on a Jasco Spectropolarimeter equipped with a recirculating water bath and Peltier temperature control unit. Temperature ramps were performed from 5 to $80^{\circ} \mathrm{C}$ with a bandwidth of $5 \mathrm{~nm}\left(1 \mathrm{~nm}\right.$ for ZJXN), ramp rate of $1{ }^{\circ} \mathrm{C} \mathrm{min}^{-1}$, equilibration time of $20 \mathrm{~s}$ and sensitivity of 100 millidegrees. Wavelength scans used the same sensitivity and bandwidth as melting runs. Spectral measurements were performed between 220 to $330 \mathrm{~nm}$ with a scan rate of $100 \mathrm{~nm} \mathrm{~min}^{-1}$. Temperature ramp profiles were fit to the Boltzmann model ${ }^{47}$, which has previously been used to determine nucleic acid melting temperatures ${ }^{48}$,

$$
\theta=\mathrm{LL}+\frac{\mathrm{UL}-\mathrm{LL}}{1+e^{\frac{T_{\mathrm{m}}-T}{a}}}
$$

where $\theta$ is the elipticity at $254 \mathrm{~nm}$ normalized to the signal change magnitude, LL and UL are the lower and upper limits of the transition, respectively, $a$ is the Hill slope, $T_{\mathrm{m}}$ is the melting temperature defined as the point of inflection of the melting curve and $T$ is the independent variable temperature.

Phi ( $\Phi)$-value analysis. $\Phi$-value analysis ${ }^{34}$ was carried out by computing $\Phi$ using equation $1\left(\Phi=\Delta \Delta G_{\mathrm{TS}}-\mathrm{WC} / \Delta \Delta G_{\mathrm{WC}-\mathrm{HG}}\right)$ where $\Delta \Delta G_{\mathrm{TS}}-\mathrm{WC}=\Delta G^{\ddagger} \mathrm{WC}-\mathrm{HG}$,mut $-\Delta G^{\ddagger} \mathrm{WC}-\mathrm{HG}, \Psi-\mathrm{WT}$ and $\Delta \Delta G_{\mathrm{WC}-\mathrm{HG}}=\Delta G_{\mathrm{WC}-\mathrm{HG}, \text { mut }}-\Delta G_{\mathrm{WC}-\mathrm{HG}, \Psi-\mathrm{WT}}$ are the change in the forward free-energy barrier $\left(\Delta G^{\ddagger} \mathrm{WC}-\mathrm{HG}\right)$ and free-energy difference between WC and HG bps $\left(\Delta G_{\mathrm{WC}-\mathrm{HG}}\right)$, respectively, on introduction of one or multiple mutations (mut) as compared with 'wild-type' $(\Psi-\mathrm{WT})$. We defined $\Psi$-WT to be $A_{5}$ T4 N3 and $A_{2}$ G10 N1 for $\mathrm{A} \bullet \mathrm{T}$ and $\mathrm{G} \bullet \mathrm{C}^{+}$bps, respectively, given that they have the lowest $\Delta \mathrm{G}_{\mathrm{WC}-\mathrm{HG}}$ values. To control for systematic errors in $\Phi$ arising due to use of a ${ }^{15} \mathrm{~N} \Psi$ - WT reference resonance, we also performed $\Phi$-value analysis assigning a ${ }^{13} \mathrm{C}$ resonance as $\Psi-$ WT for A $A$ T and $\mathrm{G} \bullet \mathrm{C}^{+}$bps, respectively. In all cases we observe $\Phi$-values concentrated around $\sim 1$ consistent with a 'late' TS. Note that $\Phi \approx 0$ when $\Delta \Delta G_{\mathrm{TS}-\mathrm{wC}} \approx 0$ relative to $\Delta \Delta G_{\mathrm{WC}-\mathrm{HG}}$ implying an early WC-like TS, whereas $\Phi \approx 1$ when $\Delta \Delta G_{\mathrm{TS}-\mathrm{WC}}$ $\approx \Delta \Delta G_{\mathrm{WC}-\mathrm{HG}}$ and implies a late HG-like TS. Errors for calculated $\Phi$-values for $\mathrm{A}_{6}$ $\mathrm{A} 17 \mathrm{C} 8, \mathrm{~A}_{2} \mathrm{~A} 3 \mathrm{Cl}^{\prime}, \mathrm{A}_{4} \mathrm{C} 15 \mathrm{C} 6$ and $\mathrm{A}_{5} \mathrm{G} 10 \mathrm{C} 8$ were larger than the corresponding values and thus could not be determined accurately.

\section{References}

1. Nikolova, E. N. et al. Transient Hoogsteen base pairs in canonical duplex DNA. Nature 470, 498-502 (2011).
2. Nikolova, E. N., Gottardo, F. L. \& Al-Hashimi, H. M. Probing transient Hoogsteen hydrogen bonds in canonical duplex DNA using NMR relaxation dispersion and single-atom substitution. J. Am. Chem. Soc. 134, 3667-3670 (2012).

3. Nikolova, E. N., Goh, G. B., Brooks, 3rd C. L. \& Al-Hashimi, H. M. Characterizing the protonation state of cytosine in transient G.C Hoogsteen base pairs in duplex DNA. J. Am. Chem. Soc. 135, 6766-6769 (2013).

4. Palmer, 3rd A. G. Chemical exchange in biomacromolecules: past, present, and future. J. Magn. Reson. 241, 3-17 (2014).

5. Sekhar, A. \& Kay, L. E. NMR paves the way for atomic level descriptions of sparsely populated, transiently formed biomolecular conformers. Proc. Natl Acad. Sci. USA 110, 12867-12874 (2013)

6. Hansen, A. L., Nikolova, E. N., Casiano-Negroni, A. \& Al-Hashimi, H. M. Extending the range of microsecond-to-millisecond chemical exchange detected in labeled and unlabeled nucleic acids by selective carbon R(1rho) NMR spectroscopy. J. Am. Chem. Soc. 131, 3818-3819 (2009).

7. Hoogsteen, K. The structure of crystals containing a hydrogen-bonded complex of 1-methylthymine and 9-methyladenine. Acta Crystallogr. 12, 822-823 (1959).

8. Nikolova, E. N. et al. A historical account of hoogsteen base-pairs in duplex DNA. Biopolymers 99, 955-968 (2013).

9. Rice, P. A., Yang, S., Mizuuchi, K. \& Nash, H. A. Crystal structure of an IHFDNA complex: a protein-induced DNA U-turn. Cell 87, 1295-1306 (1996).

10. Aishima, J. et al. A Hoogsteen base pair embedded in undistorted B-DNA. Nucleic Acids Res. 30, 5244-5252 (2002).

11. Patikoglou, G. A. et al. TATA element recognition by the TATA box-binding protein has been conserved throughout evolution. Genes Dev. 13, 3217-3230 (1999).

12. Kitayner, M. et al. Diversity in DNA recognition by p53 revealed by crystal structures with Hoogsteen base pairs. Nat. Struct. Mol. Biol. 17, 423-429 (2010).

13. Bohnuud, T. et al. Computational mapping reveals dramatic effect of Hoogsteen breathing on duplex DNA reactivity with formaldehyde. Nucleic Acids Res. 40, 7644-7652 (2012).

14. Yang, W. Structure and mechanism for DNA lesion recognition. Cell Res. 18, 184-197 (2008).

15. Yang, H., Zhan, Y., Fenn, D., Chi, L. M. \& Lam, S. L. Effect of 1-methyladenine on double-helical DNA structures. FEBS Lett. 582, 1629-1633 (2008).

16. Nair, D. T., Johnson, R. E., Prakash, S., Prakash, L. \& Aggarwal, A. K. Replication by human DNA polymerase-iota occurs by Hoogsteen base-pairing. Nature 430, 377-380 (2004).

17. Makarova, A. V. \& Kulbachinskiy, A. V. Structure of human DNA polymerase iota and the mechanism of DNA synthesis. Biochemistry (Moscow) 77, 547-561 (2012).

18. Harris, R. C. et al. Opposites attract: shape and electrostatic complementarity in protein-DNA complexes. in Innovations in Biomolecular Modeling and Simulations Vol 2 (ed. Schlick, T.) 53-80 (Royal Soc Chemistry, 2012).

19. Rohs, R. et al. The role of DNA shape in protein-DNA recognition. Nature 461, 1248-1253 (2009)

20. Johnson, R. E., Prakash, L. \& Prakash, S. Biochemical evidence for the requirement of Hoogsteen base pairing for replication by human DNA polymerase iota. Proc. Natl Acad. Sci. USA 102, 10466-10471 (2005).

21. Liu, K., Miles, H. T., Frazier, J. \& Sasisekharan, V. A novel DNA duplex. A parallel-stranded DNA helix with Hoogsteen base pairing. Biochemistry 32, 11802-11809 (1993).

22. Abrescia, N. G., Gonzalez, C., Gouyette, C. \& Subirana, J. A. X-ray and NMR studies of the DNA oligomer d(ATATAT): Hoogsteen base pairing in duplex DNA. Biochemistry 43, 4092-4100 (2004).

23. Abrescia, N. G., Thompson, A., Huynh-Dinh, T. \& Subirana, J. A. Crystal structure of an antiparallel DNA fragment with Hoogsteen base pairing. Proc. Natl Acad. Sci. USA 99, 2806-2811 (2002).

24. Cubero, E., Luque, F. J. \& Orozco, M. Theoretical study of the HoogsteenWatson-Crick junctions in DNA. Biophys. J. 90, 1000-1008 (2006).

25. Cubero, E., Abrescia, N. G., Subirana, J. A., Luque, F. J. \& Orozco, M. Theoretical study of a new DNA structure: the antiparallel Hoogsteen duplex. J. Am. Chem. Soc. 125, 14603-14612 (2003).

26. Ughetto, G. et al. A comparison of the structure of echinomycin and triostin A complexed to a DNA fragment. Nucleic Acids Res. 13, 2305-2323 (1985).

27. Gilbert, D. E., van der Marel, G. A., van Boom, J. H. \& Feigon, J. Unstable Hoogsteen base pairs adjacent to echinomycin binding sites within a DNA duplex. Proc. Natl Acad. Sci. USA 86, 3006-3010 (1989).

28. Schwartz, T., Rould, M. A., Lowenhaupt, K., Herbert, A. \& Rich, A. Crystal structure of the Zalpha domain of the human editing enzyme ADAR1 bound to left-handed Z-DNA. Science 284, 1841-1845 (1999).

29. Bothe, J. R., Lowenhaupt, K. \& Al-Hashimi, H. M. Sequence-specific B-DNA flexibility modulates Z-DNA formation. J. Am. Chem. Soc. 133, 2016-2018 (2011). 
30. Ha, S. C., Lowenhaupt, K., Rich, A., Kim, Y. G. \& Kim, K. K. Crystal structure of a junction between B-DNA and Z-DNA reveals two extruded bases. Nature 437, 1183-1186 (2005).

31. SantaLucia, Jr J., Allawi, H. T. \& Seneviratne, P. A. Improved nearest-neighbor parameters for predicting DNA duplex stability. Biochemistry 35, 3555-3562 (1996).

32. Coman, D. \& Russu, I. M. A nuclear magnetic resonance investigation of the energetics of basepair opening pathways in DNA. Biophys. J. 89, 3285-3292 (2005).

33. Gueron, M., Kochoyan, M. \& Leroy, J. L. A single mode of DNA base-pair opening drives imino proton exchange. Nature 328, 89-92 (1987).

34. Fersht, A. R., Matouschek, A. \& Serrano, L. The folding of an enzyme. I. Theory of protein engineering analysis of stability and pathway of protein folding. J. Mol. Biol. 224, 771-782 (1992).

35. Neudecker, P., Zarrine-Afsar, A., Davidson, A. R. \& Kay, L. E. Phi-value analysis of a three-state protein folding pathway by NMR relaxation dispersion spectroscopy. Proc. Natl Acad. Sci. USA 104, 15717-15722 (2007).

36. Wang, J. DNA polymerases: Hoogsteen base-pairing in DNA replication? Nature 437, E6-E7 discussion E7 (2005)

37. Honig, B. \& Rohs, R. Biophysics: flipping Watson and Crick. Nature 470, 472-473 (2011)

38. Ronning, D. R. et al. Active site sharing and subterminal hairpin recognition in a new class of DNA transposases. Mol. Cell 20, 143-154 (2005).

39. Wang, A. H. et al. The molecular structure of a DNA-triostin A complex. Science 225, 1115-1121 (1984).

40. Zhang, Y., Xi, Z., Hegde, R. S., Shakked, Z. \& Crothers, D. M. Predicting indirect readout effects in protein-DNA interactions. Proc. Natl Acad. Sci. USA 101, 8337-8341 (2004).

41. Zimmer, D. P. \& Crothers, D. M. NMR of enzymatically synthesized uniformly 13C15N-labeled DNA oligonucleotides. Proc. Natl Acad. Sci. USA 92, 3091-3095 (1995).

42. Pelupessy, P., Chiarparin, E. \& Bodenhausen, G. Excitation of selected proton signals in NMR of isotopically labeled macromolecules. J. Magn. Reson. 138, 178-181 (1999).

43. Delaglio, F. et al. Nmrpipe-a multidimensional spectral processing system based on unix pipes. J. Biomol. NMR 6, 277-293 (1995).

44. Spyracopoulos, L. A suite of mathematica notebooks for the analysis of protein main chain 15N NMR relaxation data. J. Biomol. NMR 36, 215-224 (2006).
45. Miloushev, V. Z. \& Palmer, 3rd A. G. R(1rho) relaxation for two-site chemical exchange: general approximations and some exact solutions. J. Magn. Reson. 177, 221-227 (2005).

46. Akaike, H. A new look at the statistical model identification. IEEE Trans. Aut Contr. 19, 716-723 (1974).

47. Schulz, M. N., Landstrom, J. \& Hubbard, R. E. MTSA-a Matlab program to fit thermal shift data. Anal. Biochem. 433, 43-47 (2013).

48. Doktycz, M. J., Morris, M. D., Dormady, S. J., Beattie, K. L. \& Jacobson, K. B. Optical melting of 128 octamer DNA duplexes: effects of base pair location and nearest neighbors on thermal stability. J. Biol. Chem. 270, 8439-8445 (1995).

\section{Acknowledgements}

We thank Dr Vivekanandan Subramanian for maintenance of the NMR instrument. We gratefully acknowledge Professor Ari Gafni for access to the CD instrument and Dr Joseph Schauerte for maintenance of the CD instrument. This work was supported by NIH grant GM089846 awarded to H.M.A.-H.

\section{Author contributions}

H.S.A., F.L.G. and H.M.A.-H. conceived the idea; H.S.A., F.L.G. and E.N.N. prepared samples and measured NMR data; H.S.A. carried out the data analysis with help from F.L.G., E.N.N. and H.M.A.-H.. H.S.A. and H.M.A.-H. wrote the manuscript with help from F.L.G. and E.N.N.

\section{Additional information}

Supplementary Information accompanies this paper at http://www.nature.com/ naturecommunications

Competing financial interests: The authors declare no competing financial interests

Reprints and permission information is available online at http://npg.nature.com/ reprintsandpermissions/

How to cite this article: Alvey, H. S. et al. Widespread transient Hoogsteen base pairs in canonical duplex DNA with variable energetics. Nat. Commun. 5:4786 doi: 10.1038/ ncomms5786 (2014) 